\title{
SYMPTOMS OF CONGESTIVE (CLOSED-ANGLE)* GLAUCOMA OCCURRING IN EYES WITH A WIDE CHAMBER ANGLE $\dagger$
}

\author{
BY \\ P. L. BLAXTER \\ Manchester
}

THOUGH it has been acknowledged since the beginning of the 20th century that primary glaucoma manifests itself in two distinct ways, earlier texibooks on the subject (Elliot, 1918; Henderson, 1910) do not recognize these as separate conditions but merely as variations resulting from differences in the degree of reaction to the same constant pathological factor. Thus, whilst Elliot describes the non-congestive attack of glaucoma with its characteristic lack of subjective symptoms, he states that this can pass at any time into a congestive phase.

Modern methods of examination, and especially the use of the gonioscope, have now enabled us to divide the primary glaucomas into two distinct conditions differing in aetiology, symptomatology, prognosis, and treatment.

Closed-angle glaucoma may occur at any age, but typically affects younger people than chronic simple glaucoma, especially when congestive attacks apparently due to increase in the size of the lens are discounted. Usually the symptoms are easily recognizable and these have been fully described by Miller (1952) and others; the condition manifests itself either as an acuce attack, or as a series of minor congestive episodes gradually increasing in severity until an acute attack occurs or until organic changes occur in the angle of the anterior chamber and the disease enters a chronic phase. The characteristic symptom of the early congestive attacks is misty vision and the appearance of haloes around lights, occurring typically when the patient is tired or worried, in the evening, especially after watching the cinema or television, or occasionally after reading (Higgitt and Smith, 1955).

Simple glaucoma, on the other hand, is a disease of insidious onset, seldom producing any subjective symptoms in the early stages. It can occur at any age but is most common in patients over 60 . Haloes are said to occur only very rarely in simple glaucoma and then only when the disease is in a late stage, although Sugar (1951) states that he has occasionally found them in cases of early simple glaucoma. In 99 patients Miller (1952) found that eleven advanced cases had haloes but none had them as a presenting symptom. In Miller's series no patient suffering from glaucoma simplex complained of intermittent attacks of misty vision.

*This term was recommended at the International Congress in New York to describe the condition previously referred to as congestive glaucoma or narrow-angle glaucoma (Duke-Elder, 1954).

+ Received for publication July 4, 1955. 
The reports of gonioscopy findings have further strengthened the division between the two types of glaucoma. The angle in simple glaucoma is usually medium or wide and the depth of the anterior chamber normal. This, however, is not always the case, as the angle can be any width varying from wide to narrow as can the angle in the normal eye. The term "wideangle" glaucoma advocated by Barkan (1938) is thus misleading, and the term " normal angle width" should be used for glaucoma simplex should a gonioscopic classification of glaucoma be desired. In 99 cases of glaucoma simplex, Miller found that the angles had been classified as narrow in twenty, and medium-broad in 75 (the remainder being unclassified).

Closed-angle glaucoma is associated, almost invariably, with a narrow chamber angle and a shallow anterior chamber. Miller found that of 81 cases of closed-angle glaucoma 61 had narrow angles, and Higgitt (1954) found that in all of 122 eyes with closed-angle glaucoma the angle was narrow. Sugar (1951) considers that an angle narrow enough to become blocked is the essential feature of congestive glaucoma, and that if gonioscopy fails to reveal this the diagnosis is excluded. He further points out that whilst a narrow angle is usually associated with a shallow anterior chamber, this is not invariably so, and that therefore cases of glaucoma can only be classified by gonioscopy as examination of the periphery of the cornea with the ordinary slit lamp is not reliable. I personally feel that the importance of these remarks cannot be over-emphasized.

The response of the two types of glaucoma to provocative tests also helpso to accentuate their essential differences.

It is not easy to assess the value of the water-drinking test from reports in? the literature, as the results are conflicting, and it is not always clearly stated what types of glaucoma are being considered, not whether they are in an early or advanced stage of the disease. In my experience this test, when performed on eyes of which the initial tension is normal, will only give positive results when there is an obstruction to aqueous outflow. These consist of cases of simple glaucoma or cases of chronic closed-angle glaucoma in which there are organic changes in the angle. The test is of no value in cases of closed-angle glaucoma between attacks when the angle is open.

The dark-room test, on the other hand, is essentially a test for closed-angle glaucoma. Higgitt (1954) found that the average rise after 1 hour in the dark was $2.1 \mathrm{~mm}$. $\mathrm{Hg}$ (Schiötz), and that cases of simple glaucoma gave the same figure, although there was a greater range of changes in tension when individual eyes were considered. However, in 91 cases, only two gave a rise of over $10 \mathrm{~mm}$. $\mathrm{Hg}$, the maximum being $14 \mathrm{~mm}$. $\mathrm{Hg}$.

In 122 eyes with closed-angle glaucoma in all of which the angles were narrow, the average rise was $16 \mathrm{~mm} . \mathrm{Hg}$ and the highest individual rise $64 \mathrm{~mm}$. $\mathrm{Hg}$; this test produced 78 per cent. of positive results.

The mydriatic test also will only produce a rise in tension in closed-angle 
glaucoma. The pattern of the phasic variations of the ocular tension again is different in the two conditions. In chronic simple glaucoma there appears to be an exaggeration of the normal diurnal variation so that changes in tension follow clear-cut patterns (Langley and Swanljung, 1951) changing little from day to day, but showing a gradual increase in their range and the height of the base line until the tension becomes permanently raised above the normal limit.

In the early stages of closed-angle glaucoma, the ocular tension is completely normal between attacks which at first are usually of short duracion and occur at long and irregular intervals. It should be remembered in this respect that these sudden rises in tension can, we believe, be produced by worry, so that if this occurs when a patient attends a clinic for a provocative test, and a positive result is obtained, the increase in tension may be due not to the test itself but to anxiety. It must also be pointed out, however, that sudden rises due to this cause do not appear to occur in chronic simple glaucoma, so that if such a rise is obtained it may give a false impression as to the efficiency of the test, but will still confirm the diagnosis of closed-angle glaucoma. This point will be referred to again when discussing the individual cases.

Finally, the recent development of tonometry and similar massage tests has done much to accentuate the essential differences in the two conditions, and to give a clue to the aetiology of simple glaucoma, just as gonioscopy has assisted the study of closed-angle glaucoma. These tests indicate that an obstruction to aqueous outflow is present in at least a very high proportion of cases of simple glaucoma even in the early stages.

All the above facts have given us a clearer understanding of the aetiology of glaucoma, at least as far as the immediate cause of the increase in tension in adult glaucoma is concerned.

From the evidence available, it seems probable that simple glaucoma is a condition in which there is a permanent embarrassment to the outflow of aqueous so that the effect of the vascular changes which we believe to cause the normal diurnal variation in intra-ocular tension becomes exaggerated in the glaucomatous eye, producing phases of pathologically high tension at certain times of the day. As the degree of obstruction progresses, so the periods of raised tension increase in magnitude and duration until finally the tension is permanently raised. The lack of gonioscopic evidence of any change in the filtration angle suggests that this obstruction is sited in the intrascleral vessels draining Schlemm's canal.

Closed-angle glaucoma is usually associated with a narrow angle, and attacks are believed to be due to sudden episodes during which this angle becomes blocked by the iris root. Whether the block is caused by changes in the ciliary body or iris root or by a primary pupillary block as suggested by Barkan is still a matter of conjecture.

If one accepts these facts on principle, it follows that the treatment of 
simple glaucoma, either medical or surgical, depends upon increasing the facility of aqueous outflows from Schlemm's canal either by the use of miotics to lower the episcleral venous pressure, by the surgical construction of new drainage channels, or by diminishing the secretion from the ciliary body.

In closed-angle glaucoma the attacks of high tension can be avoided by preventing the root of the iris from closing the angle. This may be done by miotics or with a basal iridectomy, either peripheral or complete. It is only when permanent changes due to peripheral synechiae have occurred in the angle that a filtering operation will be necessary.

Because there is this clear-cut difference between simple and closed-angle glaucoma it is important to be certain of the type of glaucoma we are dealing with before deciding upon our method of treatment. The description of the mode of onset of the two conditions makes this at first sight a relatively simple process, but whilst it is true to say that attacks of haloes and misty vision are symptomatic of narrow-angle glaucoma, these symptoms can also occur in the presence of a wide or medium angle, and unless we appreciate this fact we may be mislead into performing simple iridectomies on patients in whom the operation has no chance of success.

As already pointed out, the apparent depth of the anterior chamber is not an infallible guide to the problem, and only after examination with the gonioscope can an accurate diagnosis be made.

\section{Present Investigations}

The cases to be discussed, all of which presented with typical symptoms of closed-angle glaucoma, comprise only a very small proportion of those seen at the glaucoma clinic, but I feel it worth while to record them as their recognition may prevent unnecessary or unsuccessful treatment, and may also serve as a warning against a too dogmatic conception of the glaucomas.

(1) The first type of case does not represent a new condition, as it has been known for some 50 years that uveitis can cause transient attacks of haloes and misty vision with minimal signs of inflammation (Duke-Elder, 1940), and groups of these cases have been described by Posner and Schlossman (1948) and others, under the term "glaucomato-cyclitic crises". This syndrome consists of recurrent attacks of increased tension, with blurring of vision and sometimes haloes, associated with minimal signs of anterior uveitis, which can usually be recognized only with the slit lamp. The eye is relatively white and there is usually little discomfort and no pain Theodore (1952) noted that the pupil in the affected eye is larger than that in the normal eye. The attack runs a characteristic course. The tension may rise to over $70 \mathrm{~mm}$. $\mathrm{Hg}$, but usually settles rapidly, often in a few days. The keratic precipitates are small, white, and generally few in number, but it may take about a month for them to disappear completely; it is their presence that gives the only clue to the diagnosis. The attacks 
tend to recur but in spite of this the prognosis is said to be invariably good. The tension settles spontaneously and there is no damage to the optic nerve, and consequently no necessity for drastic treatment. Cases have been reported to do well on cortisone, but a weak mydriatic is usually sufficient.

Several cases of this type have been seen at the glaucoma clinic; many were referred as cases of suspected congestive glaucoma, but this diagnosis was usually rapidly excluded by gonioscopy. Unless the patient is seen during an attack the tension will be normal and all provocative tests will be negative.

However, if the patient is told to report when attack develops the keratic precipitates will eventually be discovered.

\section{Typical Case of Posner-Schlossman Syndrome}

A male, aged 27, was first seen in March, 1952. One week previously the right vision had been blurred for 2 days. Vision in the right eye was $6 / 6$, the eye was white. The anterior chamber was of normal depth and no abnormality was detected in the anterior or posterior segments. The ocular tension was $24 \mathrm{~mm}$. Hg Schiötz. Vision in the left eye was 6/24, not improved. There was a traumatic mydriasis, and the scar of an old choroidal rupture involving the macula. Apart from this the eye was normal. The ocular tension was $18 \mathrm{~mm}$. Hg Schiötz.

He attended again in April, 1954, complaining of two further attacks of blurred vision in the right eye, the last one 10 days previously; this time the attacks were accompanied by haloes. Vision in the right eye was $6 / 6$, no abnormality could be found, a Schiötz reading was not taken at this visit but the ocular tension was recorded as being normal to fingers. The left eye had not changed from the previous visit.

The patient was referred to the glaucoma clinic as a case of suspected congestive glaucoma. Here all tests proved negative. He was found to have wide chamber angles in both eyes with no P.A.S.

The tension was never found to be above 26 in the right eye. The dark-room and water-drinking tests showed no significant rise. A bulbar pressure test showed a fall of 32 per cent. in the right eye and 34 per cent. in the left.

Although there was no evidence of glaucoma the patient was told to report again immediately should he get another attack, and he attended again in June, 1954, having had an attack of blurring in the right eye 2 days before. Again the right vision and tension were normal, but he was found to have three fine white keratic precipitates in the right eye.

A diagnosis of glaucomatocyclitic crises was made.

In September, 1954, he attended again, having had an attack of blurred vision and haloes the day before in the right eye. The visual acuity was still 6/6 and the optic disc normal. Ocular tension was $52 \mathrm{~mm}$. $\mathrm{Hg}$ Schiötz in the right eye and three fine white keratic precipitates and one large one were seen. The aqueous was clear. He was put on 0.5 per cent. atropine drops, and when he was seen a week later the tension was normal and only the large keratic precipitate remained.

(2) The second group of cases are examples of congestive symptoms occurring in relatively young patients with primary glaucoma. In none is there any gonioscopic evidence of angle-block.

Case 1.-A male dental surgeon, aged 41, attended in May, 1954, for a routine refraction and mentioned that he had been seeing occasional haloes in the evenings accompanied by slight headache. These had occurred in both eyes about once a week for the last few months, and were occasionally accompanied by slight blurring of vision. There was no family history. 
Visual Acuity.-Right eye 6/5 with $1 \mathrm{D}$ cyl. and $1 \mathrm{D}$ sph. $90^{\circ}$. Left eye $6 / 5$ with $-0 \cdot 5$ D cyl. and -2 D sph. $90^{\circ}$

The discs were normal and the visual fields full.

Although he was complaining of no symptoms at the time of examination the ocular tension was $40 \mathrm{~mm}$. $\mathrm{Hg}$ Schiötz in the right eye, and $35 \mathrm{~mm}$. $\mathrm{Hg}$ Schiötz in the left. He was referred to the glaucoma clinic.

At the clinic 3 days later the ocular tension was $27 \mathrm{~mm}$. $\mathrm{Hg}$ Schiötz in the right eye and $32 \mathrm{~mm}$. Hg Schiötz in the left eye. He had had 1 per cent. pilocarpine in both eyes 1 hour before. Gonioscopy showed the angles to be medium-wide above and below and a little narrower at the sides but definitely open. No P.A.S. were seen. Tonography gave a reading of 0.04 right and 0.03 left. The bulbar pressure test showed a fall of 10 per cent. in both eyes. He was put on 2 per cent. pilocarpine and 0.5 per cent. eserine drops three times daily.

When seen 3 weeks later he was still on miotics. The ocular tension in the right eye was $29 \mathrm{~mm}$. $\mathrm{Hg}$ and in the left $32 \mathrm{~mm}$. $\mathrm{Hg}$. Tonography was still only 0.05 in the right eye and 0.06 in the left. The discs (and fields) were still normal. He was admitted for bilateral trephines.

Case 2, a female, aged 45, attended in April, 1954, complaining of misty vision and haloes occurring in both eyes every morning for the last year. One year ago she had attended hospital where she had been put on miotics for a few days and then told she was all right. This time she was referred to the glaucoma clinic.

There was a very strong family history of glaucoma; her mother was blind with the disease, her maternal aunts and uncles all had glaucoma, her grandmother and great grandmother were blind, and her son had glaucoma (Case 3).

Visual Acuity.-6/6 in both eyes, and Jaeger 1.

The optic discs were normal and the fields full. There were no inflammatory signs but the anterior chambers were a little shallow in both eyes. Gonioscopy showed the angle in the right eye to be of medium width above and below and narrow at the sides, but although there was marked movement of the iris root on altering the light it was not sufficient to close the angle. The angle in the left eye appeared to be of medium width.

The tension was $27 \mathrm{~mm}$. Hg Schiötz in both eyes.

She attended a few mornings later when the tension was $45 \mathrm{~mm} . \mathrm{Hg}$ in the right eye but the gonioscopic appearance was unchanged.

The dark-room test performed when the initial tension was $40 \mathrm{~mm}$. $\mathrm{Hg}$ in both eyes produced no rise in tension.

The water-drinking test done when the initial tension was $29 \mathrm{~mm} . \mathrm{Hg}$ in the right eye and 27 in the left produced a rise of $10 \mathrm{~mm}$. in both eyes.

Tonography performed when the tension was normal was 0.03 in the right eye and 0.05 in the left.

She was put on 2 per cent pilocarpine drops and one month later the maximum recorded tension was $27 \mathrm{~mm} . \mathrm{Hg}$ in the right eye and $22 \mathrm{~mm} . \mathrm{Hg}$ in the left.

Case 3.-A ship's officer, aged 22 (son of Case 2), was first seen in November, 1953, complaining of haloes. He had first noticed these a year ago and they occurred on coming into a dark cabin after being on deck in bright tropical light. These haloes had lasted 20 minutes. The next attack occurred 4 months later on coming out of a cinema. They now occur whenever he goes to a theatre or cinema, and sometimes at sea, especially when under stress, such as being in charge of his ship coming into port. At this time he had had about 24 attacks in all.

Visual acuity was $6 / 5$ with $0.5 \mathrm{D}$ sph. $20^{\circ}$ in the right eye and $6 / 5$ with $-0.25 \mathrm{D}$ sph. $180^{\circ}$ in the left.

Both optic discs were normal and the visual fields full. The ocular tension was $32 \mathrm{~mm}$. Hg Schiötz. 
Gonioscopy showed the angles to be very wide. There was only slight pigmentation of the trabeculae, and no P.A.S. There was a slight "on-off" movement of the iris root, but this could not possibly have blocked the angle in any circumstances.

Tonography gave a reading of 0.09 in both eyes when the tension was $32 \mathrm{~mm}$.

On the dark-room test the right eye showed a rise of $10 \mathrm{~mm}$. $\mathrm{Hg}(17-27)$, and the left eye a rise of $20 \mathrm{~mm}$. $\mathrm{Hg}(20-40)$.

He was put on 2 per cent. pilocarpine.

When seen 1 month later he had seen no haloes. The ocular tension was $22 \mathrm{~mm}$. $\mathrm{Hg}$ Schiötz in the right eye, and $20 \mathrm{~mm}$. $\mathrm{Hg}$ in the left. The discs and fields were normal. Neither eye showed any rise in the dark on miotics.

\section{Comment}

All four of the cases described presented with symptoms which could be considered typical of congestive glaucoma, but differed from it in several essential features quite apart from the gonioscopic findings.

In the Posner-Schlossman syndrome, the rise in tension is accompanied by signs of inflammation so slight that the rise in tension seems to be out of all proportion. Because of this Theodore (1952) postulated that this condition lies between true primary glaucoma and the secondary glaucomas, in as much as the tension it not secondary to the inflammation, but both the glaucoma and the cyclitis have a common aetiology, possibly allergy.

However it is not uncommon to find that it is those very eyes in which the signs of uveitis are not gross, which produce the most intractable secondary glaucomas, and most of us can recall cases of heterochromic cyclitis or sarcoidosis that illustrate this point. It is at least possible that cyclitis, however slight the physical signs, can cause vascular changes in the uveal tract and intrascleral vessels, and these changes may lead to an increased rate of formation from the ciliary body as well as partial obstruction of aqueous outflow and thus increase in tension.

In this condition the eyes will be completely normal between attacks and all provocative tests will be negative; and, unlike the eyes of cases of closedangle glaucoma, they will be found to have normal angles. Therefore when a patient gives a history of misty vision and haloes, in which neither the gonioscopic findings nor provocative tests support the diagnosis of narrow-angle glaucoma, the possibility of glaucomatocyclitis crises must be considered.

The second group consists of cases of primary simple glaucoma; these might well have been diagnosed as narrow-angle glaucoma had it not been for the gonioscopic findings, but closer consideration reveals other essential differences, in that all these eyes had tensions above normal even between attacks, which is not the case in early closed-angle glaucoma, and tonography between the attacks showed some degree of obstruction to aqueous outflow contrary to the findings of Grant (1951) and others in closed-angle glaucoma.

There is ample evidence that these cases were in fact examples of early simple glaucoma, still with normal discs but with permanent obstruction to outflow, and yet all of them had presented with congestive symptoms although none with an acute attack. It is not intended to discuss the possible 
mechanism for the symptoms, beyond saying that it did not seem to be due to angle block. The fact that these patients were of a relatively young age may be of some significance.

Finally, the results of the dark-room test in Case 3 must be mentioned. The angles (recorded by two independent observers) were extremely wide in this case and yet the rise in tension in the dark was considerable. When repeated on another occasion the rise was only small which is not an uncommon finding in closed-angle glaucoma. One can draw no valid conclusions from a single case, but, whether the rise was due to the dark or to an emotional factor, it is fairly certain that the mechanism in this case was not angle block.

It is not intended to dispute the fact that angle block is the cause of closedangle glaucoma as we understand it; these cases appear to indicate that the belief that intermittent attacks of misty vision with or without haloes are symptoms entirely confined to congestive narrow-angle glaucoma is not entirely correct.

This differentiation is not of purely academic interest, but has a real bearing on the treatment of glaucoma. When confronted with a case we should realize that the history and appearance of the anterior chamber can be misleading, but that by gonioscopic examination an accurate diagnosis can be made and lines of treatment determined accordingly.

\section{Summary}

(1) The differences between glaucoma simplex and primary congestive glaucoma are discussed. Attention is drawn to the importance of gonioscopy in diagnosing these conditions.

(2) The Posner-Schlossman syndrome is described and illustrated with a case report.

(3) Three cases of glaucoma simplex presenting with congestive symptoms are described.

(4) The differences between these four cases and true closed-angle glaucoma are discussed.

These cases were seen at the Glaucoma Clinic of the Institute of Ophthalmology and I am grateful to Sir Stewart Duke-Elder, Director of Research, for his advice and encouragement.

\section{REFERENCES}

BARKAN, O. (1938). Amer. J. Ophthal., 21, 1099.

DUKe-ElI ER. S. (1940). “ "Text-book of Ophthalmology”, vol. 3, p. $3288 . \quad$ Kimpton, London. (1954). Personal communication.

Elliot, R. H. (1918). "Glaucoma", p. 131. Lewis, London.

Grant, W. Morton (1951). Arch. Ophthal. (Chicago), 46, 113.

Henderson, T. (1910). "Glaucoma”, pp. 124,164. Arnold, London.

Higgitt, A. C. (1954). British Journal of Ophthalmology, 38, 242.

- and SMITH, R. (1955). Ibid., 39, 103.

LANGLeY, D., and SWANLJUNG, H. (1951). Ibid., 35, 445.

Miller, S. J. H. (1952). Brit. med. J., 1, 456.

Ponser, A., and Schlossman, A. (1948). Arch. Ophthal. (Chicago), 39, 517.

SugAR, H. S. (1951). "The Glaucomas ”, p. 185. Mosby, St. Louis; Kimpton, London.

THEODORE, F. H. (1952). British Journal of Ophthalmology, 36, 207. 